\title{
DESCRIPCIÓN DE TÉCNICA SIMPLIFICADA DE NEOVAGINA. REPORTE DE 4 CASOS. CALI (COLOMBIA)
}

\section{A description of a simplified neovagina technique. A report of 4 cases from Cali, Colombia}

Gustavo Gómez-Tabares, M.D.*, Albaro José Nieto-Calvache, M.D.**

Recibido: agosto 31/09 - Aceptado: abril 26/10

\section{RESUMEN}

Introducción: la agenesia de vagina representa una malformación infrecuente cuyas principales causas son el síndrome de Rokitansky-Mayer-Kuster-Hauser y la insensibilidad a los andrógenos o síndrome de feminización testicular. Su tratamiento se enfoca a la restauración de la función sexual y la mayoría de técnicas quirúrgicas descritas se caracterizan por su complejidad y requerimiento de instrumentos especiales. El objetivo de este documento es la presentación de una técnica simplificada de neovagina.

Metodología: reporte de 4 pacientes con agenesia de vagina a las que se les realizó una neovagina a través de una modificación de la técnica de McIndoe mediante la utilización de compresa adsorbente de bacterias, con seguimiento de 8 a 18 meses.

Resultados: se intervinieron tres pacientes con síndrome Rokitansky-Mayer-Kuster-Hauser y la cuarta con síndrome de feminización testicular y cariotipo XY. En uno de los casos se evidenció ruptura de recto distal al inicio de la cirugía, complicación que se identificó y corrigió de manera primaria con adecuada evolución. El tiempo de hospitalización

* Gineco-obstetra. Especialista en Medicina Reproductiva, Unidad de Endocrinología Reproductiva, Departamento de Ginecología y Obstetricia, Hospital Universitario del Valle. Cali (Colombia).

** Residente Ginecología y Obstetricia, Universidad del Valle. Cali (Colombia). Correo electrónico: albarojosenieto@yahoo.com varió de 2 a 3 días. No hubo evidencia de complicaciones a mediano y largo plazo, y se documentó el inicio de relaciones sexuales alrededor de 40 días posteriores a la intervención.

Conclusión: la técnica quirúrgica descrita surge como una opción que hace el procedimiento más sencillo para restaurar la función sexual de mujeres con esta afección.

Palabras clave: síndrome de Rokitansky-MayerKuster-Hauser, técnica quirúrgica, neovagina.

\section{SUMMARY}

Introduction: vaginal agenesis represents a rare malformation, mostly associated with Mayer-Rokitansky-Kuster-Hauser syndrome and androgen insensitivity syndrome or testicular feminisation syndrome. Treatment focuses on restoring sexual function and most surgical techniques are characterised by their complexity and require special instruments. This paper was aimed at presenting a simplified neovagina technique.

Methodology: four cases of patients suffering vaginal agenesis are reported; they underwent a modification of the McIndoe technique by using absorbent antimicrobial barrier dressings which simplifies the procedure and reduces implementation costs. They were followed-up for 8-18 months and suitable results were obtained. 
Results: three patients suffering from MRKH syndrome were treated; the fourth one had testicular feminisation syndrome (XY karyotype). A rupture of the distal colon or rectum was seen at the start of surgery in one case; such complication was identified and corrected, presenting suitable evolution. Hospital stay varied from 2-3 days. No medium or long-term complications were presented. Sexual relationships were documented as having begun around 40 days later.

Conclusion: the surgical technique described here represents an option for restoring sexual function in women suffering from this condition.

Key words: Mayer-Rokitansky-Kuster-Hauser (MRKH) syndrome, surgical technique, neovagina.

\section{INTRODUCCIÓN}

La agenesia de vagina es una malformación poco frecuente (1 en 5.000-10.000 recién nacidas vivas). ${ }^{1,2}$ En la mayoría de ocasiones el principal síntoma de las pacientes afectadas es una alteración en su vida sexual; pueden consultar por dolor o masas pélvicas asociadas a hematómetra. Las dos principales causas son el síndrome de feminización testicular o insensibilidad a los andrógenos y el síndrome de Rokitansky-Mayer-Kuster-Hauser. Este último comprende un espectro de alteraciones embrionarias de los conductos mullerianos con agenesia parcial o total de la vagina y el útero, además de asociarse de manera frecuente con agenesia renal (40\% de los casos), ${ }^{3}$ alteraciones esqueléticas (10-12\% de los casos) ${ }^{4}$ y malformaciones auditivas $(4,5 \%) .^{5}$ Estas mujeres tienen cariotipo normal $46 \mathrm{XX}$ y función hormonal ovárica normal. Por su parte, en el síndrome de feminización testicular se encuentra cariotipo $46 \mathrm{XY}$.

El manejo quirúrgico de esta entidad se dirige a restablecer la función sexual y a la creación de un canal vaginal que haga posible la función sexual normal. Se han descrito técnicas conservadoras que consisten en la dilatación progresiva de la membrana pseudohimeneal desde el exterior, siendo útil sólo en pacientes que presenten definido el introito, ${ }^{6}$ y alrededor de 11 técnicas quirúrgicas para crear una neovagina: ${ }^{7}$ moldes plásticos con forma de oliva combinados con dispositivos de tracción para la formación de una cavidad entre la vejiga y el recto, con su epitelización desde el exterior, ${ }^{8}$ con abordaje por laparotomía o laparoscopia, ${ }^{9}$ trasplante de tejido intestinal, ${ }^{10,11}$ colgajos abdominales, pudendos ${ }^{12}$ o del muslo, injertos de piel, ${ }^{13-16}$ mucosa, peritoneo ${ }^{17}$ o amnios. ${ }^{18}$ Algunas de estas técnicas implican tiempo quirúrgico prolongado y amplia disección de tejidos (incisión abdominal y perineal, resección intestinal, disección de gran extensión del periné, entre otros procedimientos) con los riesgos incrementados de una cirugía amplia y con considerable incapacidad posquirúrgica; otras requieren el empleo de recursos tecnológicos que en nuestro medio no se encuentran disponibles en todos los hospitales, como equipos de laparoscopia y dispositivos especialmente diseñados (dispositivos de tracción, dermátomo, etcétera). La técnica utilizada por nuestro grupo incluía la disección del tabique rectovesical desde un abordaje perineal con la posterior fijación de un molde en la neovagina (figura 1), cubierto por un injerto de piel extraída de la zona glútea. ${ }^{19}$

El objetivo de la presentación de este reporte de casos es describir una variación de la técnica que la hace más simple pues se necesitan menos recursos tecnológicos y humanos, además de reducir el tiempo quirúrgico.

\section{METODOLOGÍA}

Reporte de casos de pacientes con agenesia de vagina, entre agosto de 2008 y julio de 2009, en el Hospital Universitario del Valle, centro de referencia de la región suroccidental del país. Se solicitó autorización escrita a las pacientes para la realización del procedimiento, previa descripción de la técnica a utilizar y para realizar la descripción de sus casos clínicos, manteniendo siempre la confidencialidad de sus identidades. Las pacientes recibieron evaluación hormonal, cromosómica y anatómica pélvica (ecografía pélvica, resonancia magnética nuclear o escanografía pélvica) y renal (ecografía y urografía excretora). 
Figura 1. (A)Introito vaginal previo a la cirugía donde se evidencia la ausencia de canal vaginal. (B) Incisión en introito vaginal donde se inicia la disección roma por el plano de clivaje identificado en tabique rectovesical.
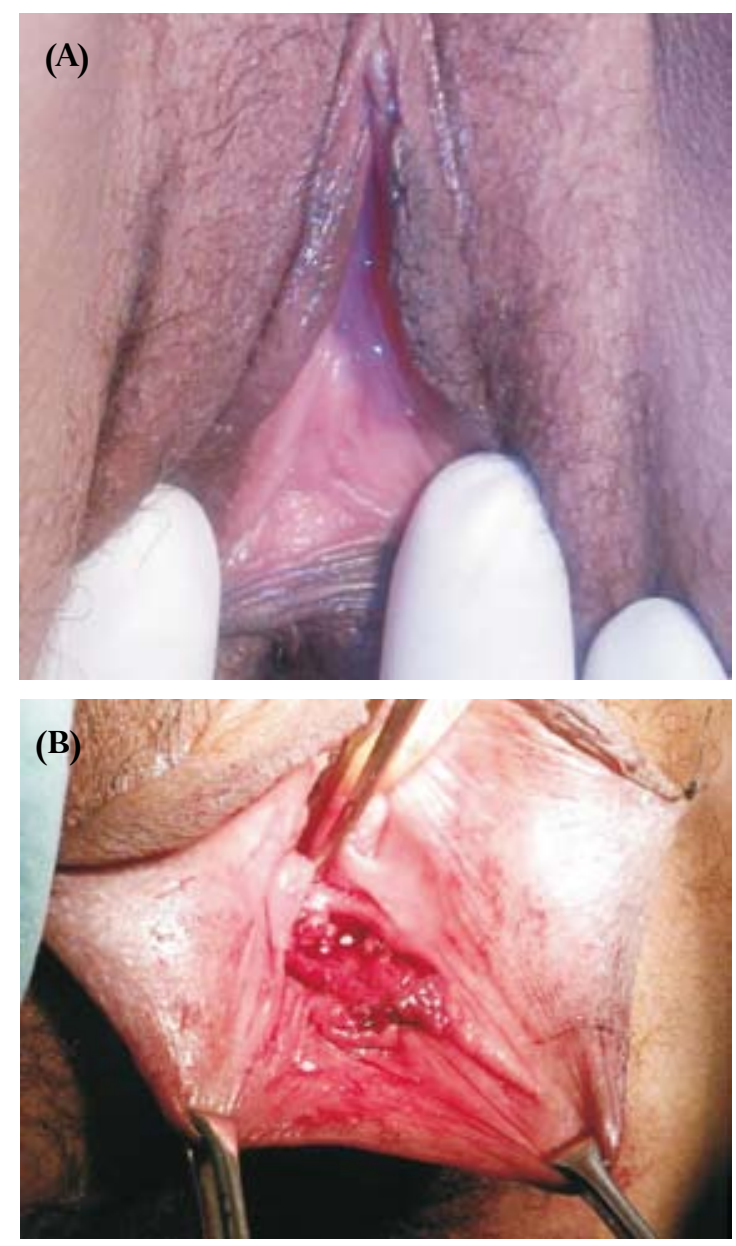

\section{DESCRIPCIÓN DE LA TÉCNICA Y DEL PROCEDIMIENTO QUIRÚRGICO}

Se describe una modificación de la técnica previamente descrita ${ }^{19}$ en la cual se reemplazó del injerto cutáneo por un material comercial (Cutisorb ${ }^{\circledR}$ Sorbact $^{\circledR}$, compresa adsorbente de bacterias, 179 x 137mm BSN medical GmbH \& co. HamburgGermany) (figura 2) que busca favorecer la reepitelización.

El manejo posquirúrgico incluyó cateterismo vesical permanente por 24-36 horas y antibióticos profilácticos durante el tiempo que se mantuviera el cateterismo. Posterior a este tiempo no se
Figura 2. (A) Preparación de molde de balso cubierto por condón y luego por compresa adsorbente. La compresa se obtiene en forma rectangular, se corta y se cose con sutura absorbible (en nuestras pacientes utilizamos poliglactina 910) a la medida del molde, inmediatamente previo a la inserción. (B) Inserción del molde en neovagina. (C) Fijación del molde mediante dispositivo diseñado con materiales disponibles en el quirófano (campo quirúrgico, gasas y esparadrapo), que permite su apertura para la defecación.
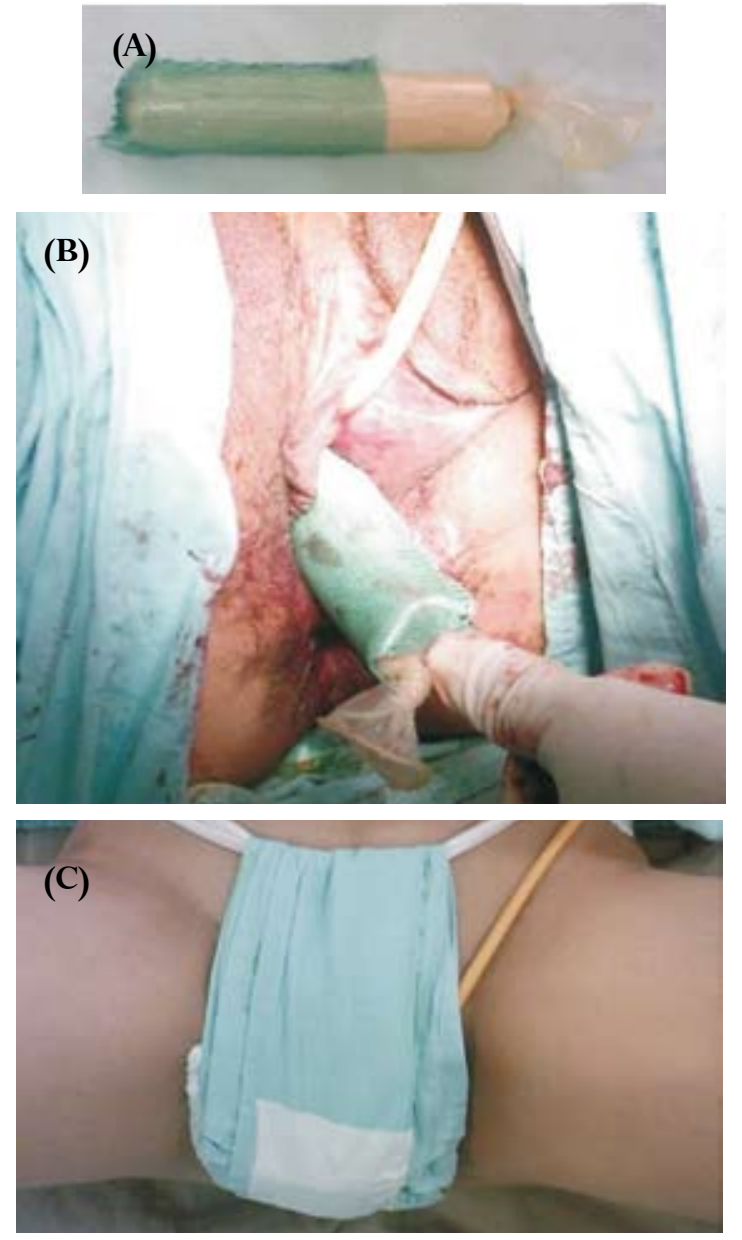

administraron antibióticos. Para el manejo del dolor se utilizaron antiinflamatorios no esteroideos endovenosos.

\section{MANEJO POSQUIRÚRGICO}

Una semana después de la cirugía se retiró el molde (figura 3) y según el grado de cicatrización de la neovagina, se colocó una segunda compresa adsorbente de bacterias o se impregnó el molde con crema estimuladora de la reepitelización (extracto 
Figura 3. (A)Retiro del molde vaginal 1 semana después del procedimiento, donde se evidencia tejido de granulación y ausencia de secreción purulenta o signos de infección. (B) Se evidencia el introito vaginal en proceso de epitelialización, inmediatamente luego de retirar el molde.
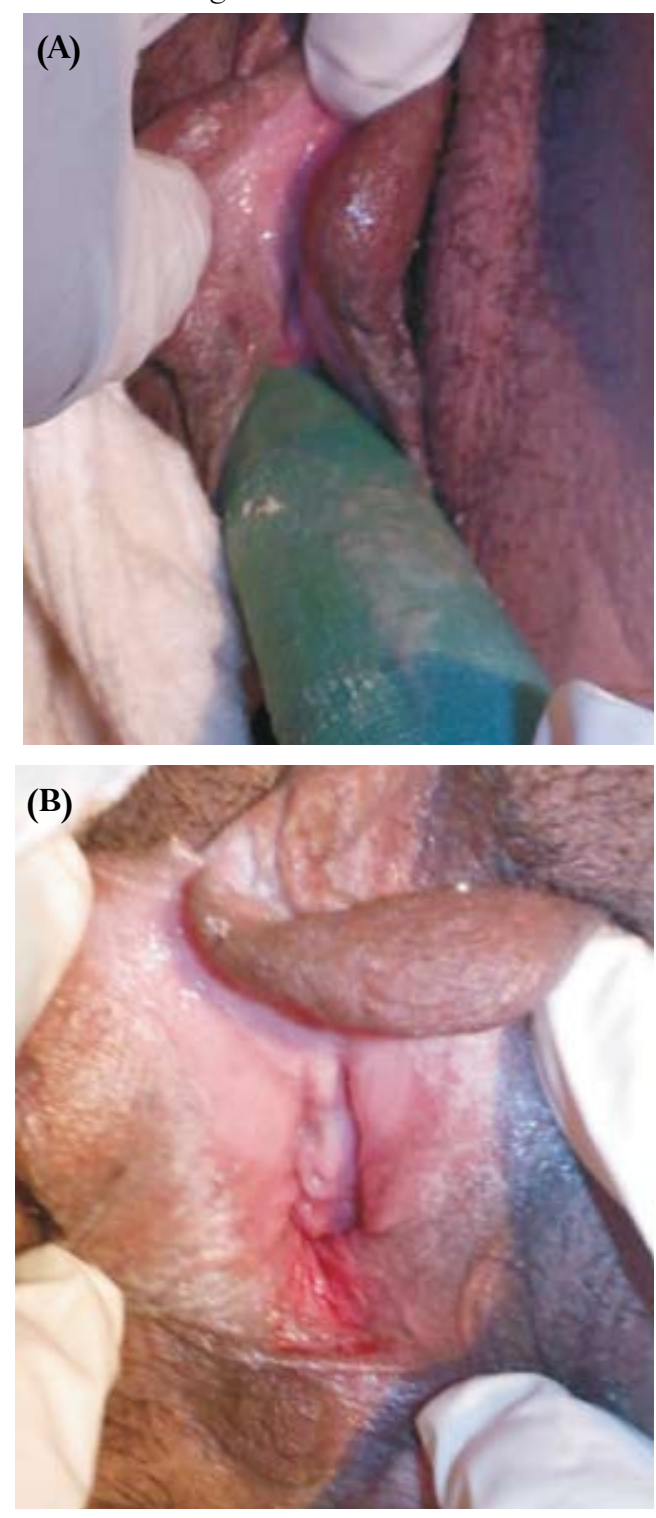

acuoso de Triticum vulgare, fitostimulina $\left.{ }^{\circledR}\right)$. También se recomendó utilizar el molde toda la noche y en el día su colocación intermitente durante al menos 2 horas los tres primeros meses. Se indicó iniciar relaciones sexuales 1 mes después de la cirugía, y si a los tres meses éstas se presentaban de manera regular, se recomendó abandonar el uso del molde, de lo contrario su utilización debía continuar por lo menos 1 hora al día por un año. ${ }^{20}$
En todos los casos se recomendó la utilización de crema de estrógenos en el introito vaginal dos veces por semana y la dilatación voluntaria con molde o por medio de interacción sexual con la pareja.

Se realizaron controles pasados 8 y 15 días, 1,2 y 8 meses. En todos los casos se realizó examen físico con medición de la profundidad y diámetro de la vagina, se interrogó sobre la frecuencia de sus relaciones sexuales y se preguntó sobre el grado de satisfacción con la cirugía. Para este último punto se utilizó una escala visual compuesta por un continuum de 1 a 5 (1, ninguna mejoría, a 5, mejoría muy grande).

\section{RESULTADOS}

Se intervinieron cuatro pacientes: tres de ellas con síndrome Rokitansky-Mayer-Kuster-Hauser y agenesia total de vagina y útero; la cuarta presentaba síndrome de feminización testicular y cariotipo XY. Las cuatro pacientes (tabla 1) exhibían desarrollo normal de características sexuales secundarias y una paciente presentaba otras malformaciones congénitas músculo-esqueléticas y renales. Una paciente estaba casada, dos tenían pareja sexual estable y la tercera no había tenido experiencia sexual previa. La duración de la cirugía varió entre 40 y 90 minutos, en uno de los casos se evidenció ruptura de recto distal al inicio de la cirugía, complicación que se identificó y corrigió de manera primaria con adecuada evolución. Se observó deambulación luego de 24-36 horas. El tiempo de hospitalización posquirúrgico tuvo un rango de 2-3 días (tabla $\mathbf{1}$ ).

No se observó retención urinaria luego de retirar la sonda vesical en ningún caso. En la visita de control ocho días posquirúrgico las pacientes referían mínima o ninguna limitación funcional y no se observó infección del sitio operatorio en ninguna de las pacientes. En visitas posteriores, no se evidenciaron complicaciones ni quejas por parte de las pacientes y se documentó el inicio de relaciones sexuales alrededor de 40 días.

A la fecha de publicación se realizó seguimiento hasta 18 meses en 3 pacientes y 8 meses en la última paciente intervenida. Se evidencia adecuada 


\section{Tabla 1. Características de pacientes intervenidas.}

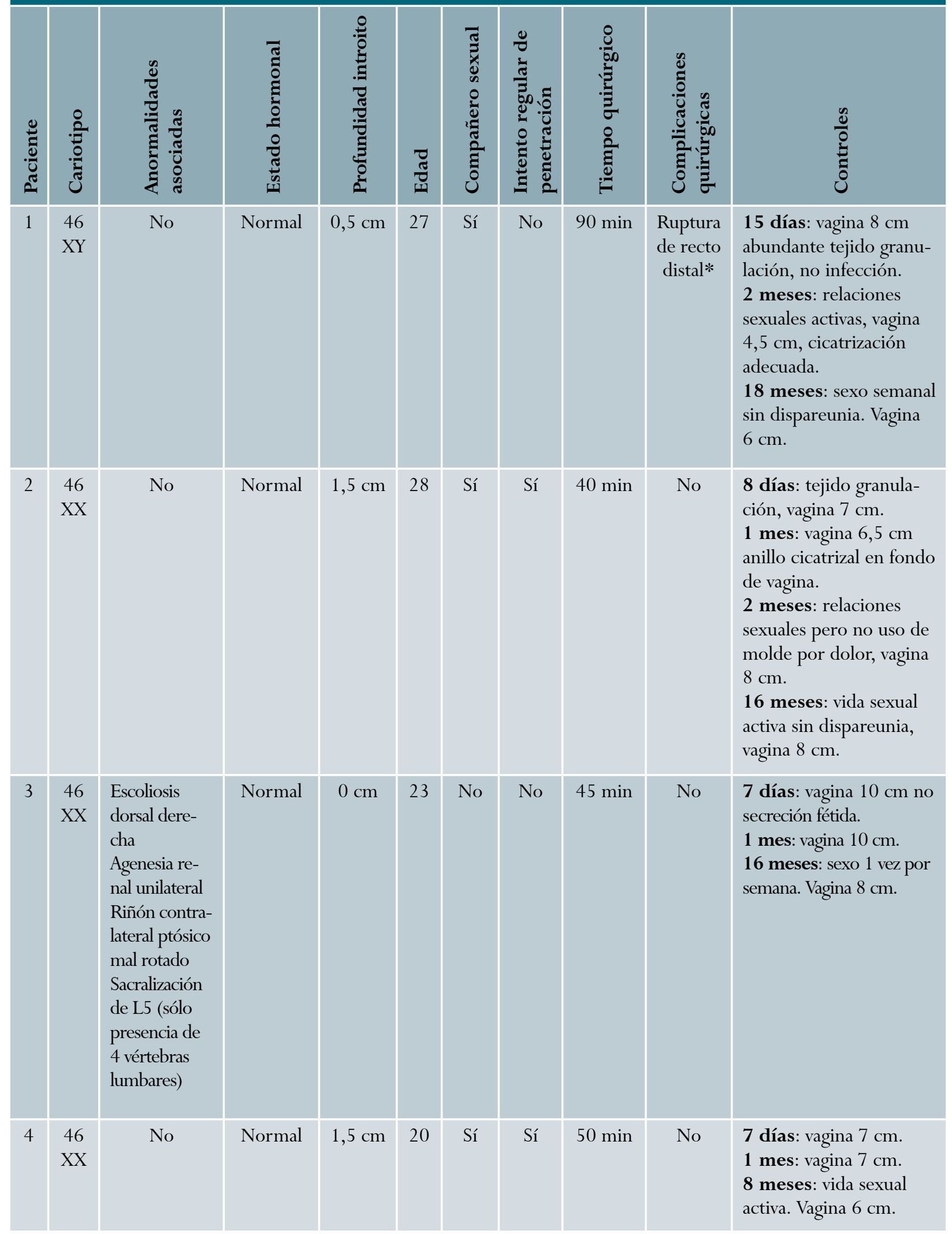

* Se identifica ruptura de $1 \mathrm{~cm}$ en la pared anterior del recto distal que se corrigió en el acto quirúrgico con sutura de catgut cromado 2-0 en 2 capas (mucosa y músculo). 
cicatrización, vagina con profundidad de 6 a $8 \mathrm{~cm}$ y vida sexual activa. Todas las pacientes refirieron estar satisfechas con los resultados de la cirugía eligiendo en la escala visual de satisfacción la máxima calificación posible.

\section{DISCUSIÓN}

Aunque la incidencia de agenesia vaginal es baja, las mujeres afectadas presentan un gran impacto psicológico y social, particularmente en su vida de pareja, lo cual ha motivado el desarrollo de múltiples métodos de tratamiento en busca de restaurar su función sexual. Muchas de las técnicas quirúrgicas publicadas son invasivas y dispendiosas, mientras otras requieren la aplicación de tecnología que en nuestro medio no está al alcance de toda la población.

La variación propuesta a la técnica de McIndoe ofrecería algunas ventajas en este sentido. La no utilización de injerto cutáneo o de mucosa hace que el procedimiento sea más rápido y sencillo, limita el requerimiento de personal e insumos adicionales para el manejo del sitio de toma del injerto y al parecer, reduce el dolor, los cuidados posquirúrgicos y el tiempo de recuperación; elimina la cicatriz permanente en el sitio donador del injerto, además deja a un lado las preocupaciones sobre su incorrecta fijación y el despegamiento al retirar el molde, así como la acumulación de fluidos entre el injerto y el tejido subyacente con las infecciones subsecuentes. Por lo tanto los insumos para su realización son mínimos de manera que podría llevarse a cabo en cualquier sala de cirugía por el personal capacitado.

Entre las principales complicaciones del procedimiento se encuentran la formación de lesión en órganos adyacentes, la formación de fístulas (que pueden estar en relación con tamaño inapropiado del molde, infección, devascularización traumática o acumulación de fluidos), infección y reestenosis de la neovagina. ${ }^{21,22}$ La limitada manipulación de tejidos, el escaso drenaje de secreciones observado con la técnica estudiada y la hemostasia selectiva durante el procedimiento quirúrgico, harían menos probable la aparición de este tipo de complicaciones y pueden ser factores contribuyentes a los adecuados resultados observados en nuestras pacientes.

Esta técnica tendría como mayor ventaja su sencillezy mínimos costos. Se hace necesario comparar esta técnica con otras técnicas mediante un ensayo clínico controlado para evaluar el comportamiento de la neovagina en los aspectos psicosexuales de calidad de vida o de satisfacción del paciente a largo plazo, mediante instrumentos previamente validados, ${ }^{11,21-24}$ para constatar los aspectos favorables atribuibles a la aplicación de esta técnica.

\section{CONCLUSIÓN}

La variación descrita de nuestra técnica quirúrgica para neovagina surge como una opción más práctica, más segura, con menos riesgo de morbilidad posquirúrgica y (aunque no se hicieron estudios de costos) aparentemente más económica para restaurar la función sexual de mujeres con agenesia de vagina.

\section{REFERENCIAS}

1. Evans PN, Poland NL, Boving RL. Vaginal malformations. Am J Obstet Gynecol 1981;141:910-20.

2. Rock JA, Azziz R. Genitalia anomalies in childhood. Clin Obstet Gynecol 1987;30:682-96.

3. Malewski AW, Czaplicki M, Kryst P. Congenital abnormality of the urinary tract in Mayer-RokitanskyKuster-Hauser syndrome. Ginekol Pol 1992;63:251-4.

4. Strubbe EH, Lemmens JA, Thijn CJ, Willemsen WN, van Toor BS. Spinal abnormalities and the atypical form of the Mayer-Rokitansky-Kuster-Hauser syndrome. Skeletal Radiol 1992;21:459-62.

5. Wulfsberg EA, Grigsby TM. Rokitansky sequence in association with the facio-auriculo- vertebral sequence: part of a mesodermal malformation spectrum? Am J Med Genet 1990;37:100-2.

6. Frank RT. The formation of an artificial vagina without operation. Am J Obstet Gynecol 1938;35:1053-5.

7. Nichols DH, Randall CL. Creation of a neovagina. En: Nichols DH, Randall CL. Vaginal surgery. 3rd ed. New York: Appleton;1989. p. 413-25.

8. Vecchietti G. Neovagina nella syndrome di RokitanskyKuster-Hauser. Attual Obstet Ginecol 1965;11:131-47. 
9. Borruto F, Chasen ST, Chervenak FA, Fedele L. The Vecchietti procedure for surgical treatment of vaginal agenesis: comparison of laparoscopy and laparotomy. Int J Gynecol Obstet 1999;64:153-8.

10. Burger RA, Riedmiller H, Knapstein PG, Friedberg V, Hohenfellner R. Ileocecal vaginal construction. Am J Obstet Gynecol 1989;161:162-7.

11. Brun J, Belleannée G, Grafeillec N, Asland AF, Brun GH. Long-term results after neovagina creation in Mayer-Rokitanski-Kuster-Hauser syndrome by Vecchietti's operation. Eur J Obstet Gynecol Reprod Biol 2002;103:168-72.

12. Sheares BH. Congenital atresia of the vagina: a technique for tunneling the space between the bladder and rectum and construction of a new vagina by a modified Wharton-technique. J Obstet Gynecol Br Emp 1960;67:24-31.

13. Alessandrescu D, Peltecu GC, Buhimschi CS, Buhimschi IA. Neocolpopoiesis with split-thickness skin graft as a surgical treatment of vaginal agenesis: retrospective review of 201 cases. Am J Obstet Gynecol 1996;175:131-8.

14. Rock JA. Surgery for anomalies of the mullerian ducts. En: Rock JA, Thompson JD, eds. TeLinde's operative gynecology. 50th ed. New York: LippincottRaven;1997. p. 687-729.

15. Fliegner JR. Congenital atresia of the vagina. Surg Gynecol Obstet 1987;175:387-91.
16. McIndoe AH, Banister JB. An operation for the cure of congenital absence of the vagina. J Obstet Gynaecol Br Empire 1938;45:490-4.

17. Rothman D. The use of peritoneum in the construction of a vagina. Obstet Gynecol 1972;40:835-8.

18. Morton KE, Dewhurst CJ. Human amnion in the treatment of vaginal malformations. Br J Obstet Gynaecol 1986;93:50-4.

19. Gómez G. Síndrome de disgenesia de conductos mullerianos: Agenesia de cuello, 2/3 superiores de vagina. Rev Colomb Obstet Ginecol 1994;45:81-3.

20. Yu K, Lin Y, Chao K. A detachable porous vaginal mold facilitates reconstruction of a modified McIndoe neovagina. Fertil Steril 2004;81:435-9.

21. Borruto F. Mayer-Rokitansky-Küster Syndrome: Vecchietti's personal series. Clin Exp Obstet Gynecol 1992;19:273-4.

22. Creatsas G. Deligeoroglou E, Makrakis E, Kontoravdis A, Papadimitriou L. Creation of a neovagina following Williams vaginoplasty and the Creatsas modification in 111 patients with Mayer-Rokitansky-Küster-Hauser syndrome. Fertil Steril 2008;90:1940-52.

23. Diener E. Subjective well-being: The science of happiness and a proposal for a national index. Am Psychol 2000;55:34-43.

24. Andersen BL, Cyranowski JM. Women's sexual selfschema. Journal of Personality and Social Psychology 1994;67:1079-100. 